Deuteron NMR relaxation, spectra, and evidence for the order-disorder phase transition in $\left(\mathrm{ND}_{4}\right)_{2} \mathrm{PtCl}_{6}$

A. Birczyński, E. E. Ylinen, M. Punkkinen, M. Prager, A. M. Szymocha, and Z. T. Lalowicz

Citation: The Journal of Chemical Physics 128, 184510 (2008); doi: 10.1063/1.2919568

View online: https://doi.org/10.1063/1.2919568

View Table of Contents: http://aip.scitation.org/toc/jcp/128/18

Published by the American Institute of Physics 


\title{
Deuteron NMR relaxation, spectra, and evidence for the order-disorder phase transition in $\left(\mathrm{ND}_{4}\right)_{2} \mathrm{PtCl}_{6}$
}

\author{
A. Birczyński, ${ }^{1}$ E. E. Ylinen, ${ }^{2}$ M. Punkkinen, ${ }^{2}$ M. Prager, ${ }^{3}$ A. M. Szymocha, ${ }^{1}$ and \\ Z. T. Lalowicz ${ }^{1, a)}$ \\ ${ }^{1}$ H. Niewodniczański Institute of Nuclear Physics of PAS, 31-342 Kraków, Poland \\ ${ }^{2}$ Wihuri Physical Laboratory, Department of Physics, University of Turku, FI-20014 Turku, Finland \\ ${ }^{3}$ Institut für Festkörperforschung des Forschugszentrums Jülich, D-52425 Jülich, Germany
}

(Received 1 February 2008; accepted 11 April 2008; published online 14 May 2008)

\begin{abstract}
Deuteron NMR relaxation and spectra were studied at the resonance frequency of $46 \mathrm{MHz}$ in polycrystalline $\left(\mathrm{ND}_{4}\right)_{2} \mathrm{PtCl}_{6}$ between $300-5 \mathrm{~K}$. The relaxation rate maximum near $50 \mathrm{~K}$ is about $53 \%$ smaller than the calculated maximum related to $120^{\circ}$ rotations about the threefold symmetry axes of the ammonium ion. The difference is explained by assuming for a N-D vector a total of 24 equilibrium directions, which in groups of six deviate from the nearest $\mathrm{Pt}-\mathrm{N}$ vector by a certain angle $\Theta$. So-called limited jumps between the directions of each group take place much more frequently than the large-angle rotations, thus rendering a fraction of the deuteron quadrupole coupling ineffective in relaxation. A motional model is presented, which takes into account both these motions simultaneously. A comparison with experimental data leads to $\Theta=26.0^{\circ}$, in reasonable agreement with earlier neutron diffraction data. A sharp decrease found in the relaxation rate at the order-disorder phase transition temperature of $27.2 \mathrm{~K}$ is related to the fact that one of the six equilibrium directions becomes preferred. This leads to a formation of ordered domains, in which the active motion driving the relaxation is $120^{\circ}$ rotations. Two components in the spectra found below $55 \mathrm{~K}$ are related to domains (broad) and transition regions between domains (narrow). Reasons for the nonexponentiality observed below $20 \mathrm{~K}$ are discussed, the most likely explanation being that limited jumps dominate within transition regions and make the corresponding deuterons relax faster than those in domains. (C) 2008 American Institute of Physics.
\end{abstract}

[DOI: 10.1063/1.2919568]

\section{INTRODUCTION}

Deuteration induced phase transitions (PTs) were discovered in a number of compounds. ${ }^{1}$ Completely or partly deuterated ammonium hexachlorometallates provide several examples of such an effect. It was first observed in $\left(\mathrm{ND}_{4}\right)_{2} \mathrm{PdCl}_{6}$ by neutron diffraction, ${ }^{2}$ but subsequent studies by chlorine NMR and especially calorimetric experiments have detected it and explored its properties also in corresponding $\mathrm{Pt},{ }^{3} \mathrm{Se}, \mathrm{Te},{ }^{4}$ and $\mathrm{Pb}^{5}$ compounds. The nature of PTs was explained in detail in review papers by Weir and Westrum $^{6}$ and Matsuo. ${ }^{7}$ There usually exists a sequence of crystal phases on decreasing temperature and increasing deuteration. ${ }^{4}$ A displacive type of PT may be caused by a rotation of the octahedral hexachlorometallate cation by an angle from the usual equilibrium position with the $\mathrm{Me}-\mathrm{Cl}$ bonds along the edges of the unit cell. Prandl and co-workers 8,9 applied neutron diffraction and report that there are six equal minima in the rotational potential corresponding roughly to $\pm 25^{\circ}$ rotations of $\mathrm{ND}_{4}^{+}$around the $X, Y$, and $Z$ axes in the unit-cell fixed frame of ammonium hexachlorostannate.

The chlorine quadrupole resonance frequency and also its spin-lattice relaxation very sensitively show even small

\footnotetext{
a) Author to whom correspondence should be addressed. TEL.: +48 126628 259. FAX: $+48 \quad 126628$ 458. Electronic mail: zdzislaw.lalowicz@ifj.edu.pl.
}

changes in the crystal structure. In addition to the experiments on $\mathrm{Se}$ and Te hexachlorometallates mentioned above $\mathrm{e}^{4}$ the chlorine resonance was studied also in $\left(\mathrm{ND}_{4}\right)_{2} \mathrm{PtCl}_{6}$. ${ }^{10}$ Additional information can be obtained by deuteron NMR resonance and relaxation studies. Spectral studies on Te and $\mathrm{Pb}$ compounds yield the temperature dependence of the $\mathrm{ND}_{4}^{+}$ tunneling splitting and reveal the effect of the order-disorder transition. ${ }^{11,12}$ Partial deuteration was observed to play a dominant role in the spin-lattice relaxation of both the deuterons and protons in ammonium hexachlorotellurate. ${ }^{13,14}$

Deuteron NMR studies provide evidence for diverse mobility in the ordered phase. Ammonium ions inside ordered domains are immobile in $\left(\mathrm{ND}_{4}\right)_{2} \mathrm{PtCl}_{4}$ at low temperatures. However, about $10 \%$ of ions remain highly mobile at the domain walls. ${ }^{15}$ Alternatively, in the case of $\left(\mathrm{ND}_{4}\right)_{2} \mathrm{TeCl}_{6}$, two tunneling frequencies of ammonium ions were found, 2.2 and $0.5 \mathrm{MHz}$, attributed to domain walls and bulk domains, respectively. ${ }^{11}$

In this study we have carried out careful experiments on the deuteron NMR line shape and spin-lattice relaxation in $\left(\mathrm{ND}_{4}\right)_{2} \mathrm{PtCl}_{6}$ from room temperature down to $5 \mathrm{~K}$. Special attention was paid to temperatures around the order-disorder transition at $27.2 \mathrm{~K}^{6}$

The interpretation of the results requires different aspects of molecular mobility as follows:

Ammonium reorientation by symmetry jumps. 
(ii) Limited jumps (LJs) between the six equilibrium orientations of a N-D bond near a certain Pt-N direction. Such orientations are obtained via a rotation of ammonium ion by an angle $\varphi$ about the fourfold axis of the high-temperature cubic unit cell. ${ }^{16,17}$

(iii) The ordering of the $\mathrm{ND}_{4}^{+}$, which seems to start, to some degree, already well above the transition temperature.

(iv) Incoherent tunneling at lower temperatures.

A short discussion on rotational states is given first as there is a very low potential for $\mathrm{ND}_{4}^{+}$reorientation in $\left(\mathrm{ND}_{4}\right)_{2} \mathrm{PtCl}_{6}$. Then a model is presented for the deuteron spin-lattice relaxation via the deuteron quadrupole interaction, modulated by reorientations and LJ. Experimental results are presented next and interpreted in terms of this model and the three different aspects of the molecular mobility. The reduced efficiency of reorientations in the relaxation, already observed many times before, is explained by a partial averaging of the quadrupole interaction by LJ.

\section{THEORETICAL CONSIDERATIONS}

\section{A. Rotational states and tunneling splitting}

The hindering potential for ammonium reorientations is generally rather weak in ammonium hexachlorometallates. The weakest potential was obtained for hexachloropalladate and next weakest for $\left(\mathrm{NH}_{4}\right)_{2} \mathrm{PtCl}_{6}$ and respectively, for the corresponding deuterated compounds. ${ }^{18}$ As a consequence, the tunneling frequency between the $A$ and $3 T$ sublevels of the ground rotational state, $\nu_{\mathrm{TA}}$, equals $7.0 \mathrm{GHz}$ in the protonated sample at liquid helium temperatures. ${ }^{19}$ The symbols $A$ and $T$, together with $E^{a}$ and $E^{b}$, refer to the irreducible representations of the tetrahedral point group. The rotational wave functions transform according to these irreducible representations. In the case of a potential having a tetrahedral or higher symmetry all the three $T$ levels are degenerate, but the degeneracy is lifted for lower symmetries.

The rotational levels for a one-dimensional rotor like the methyl group can be solved from the Mathieu equation, either approximately in a closed form ${ }^{20,21}$ or exactly numerically. ${ }^{22}$ For threefold rotors like $\mathrm{CH}_{4}, \mathrm{NH}_{4}^{+}$and their deuterated counterparts no such relatively simple method is available. However, the tunneling splitting of both the oneand three-dimensional rotors depend in principle in the same way on the hindering potential. This assumption, together with the approximate closed-form solution for the Mathieu equation, was used to evaluate the tunneling frequency from the value of the hindering potential, or actually from the activation energy of the ammonium reorientations. ${ }^{22}$ Unfortunately, even a small difference in the activation energy causes a big change in $\nu_{\mathrm{TA}}$. The present data for the deuteron relaxation in $\left(\mathrm{ND}_{4}\right)_{2} \mathrm{PtCl}_{6}$ give the activation energy $E_{a}$ $=3.4 \mathrm{~kJ} / \mathrm{mol}$, which leads to the value $\nu_{\mathrm{TA}}=42.0 \mathrm{MHz}$ at liquid helium temperatures. Calculations on the temperature dependence of $\nu_{\mathrm{TA}}$ predict its reduction to zero at about $38 \mathrm{~K}$. Assuming for $\mathrm{ND}_{4}^{+}$the same potential as for $\mathrm{NH}_{4}^{+}$in the protonated compound, the result $\nu_{\mathrm{TA}}=204 \mathrm{MHz}$ was obtained. $^{22}$ Thus the activation energy appears to be higher in the deuterated compound.
The symmetry of the spin function of the four deuterons is related to the symmetry of the rotational wave function, since the total wave function (being the product of the rotational and spin functions) must remain unchanged under the symmetry rotations of $\mathrm{ND}_{4}^{+}$. Therefore, only the total spins $I=0,2,4$ are possible with the rotational functions of the $A$ symmetry, $I=1,2,3$ with the $T$ symmetry and $I=0,2$ with the $E\left(=E^{a}+E^{b}\right)$ symmetry. This coupling has certain consequences concerning the deuteron NMR spectrum and relaxation.

\section{B. Relaxation via reorientation and limited jumps}

Ammonium motion by $120^{\circ}$ rotations, or by the equivalent part of incoherent tunneling at low temperatures, is the common mechanism of spin-lattice relaxation. LJs were proposed to become important at low temperatures. ${ }^{16,17}$ Here we present a model, which includes both motions simultaneously. The model assumes that all the tunneling frequencies are clearly smaller than the deuteron resonance frequency $\nu_{0}=46 \mathrm{MHz}$, what considerably simplifies the derivation but also limits its applicability to some extent. At first the equilibrium positions of the deuterons are defined. The edges of the high-temperature cubic unit cell are taken as the $X, Y$, and $Z$ axes of the molecular coordinate frame. The four $\mathrm{Pt}-\mathrm{N}$ directions are parallel to the body diagonals $\left[\begin{array}{lll}-1 & -1 & 1\end{array}\right],\left[\begin{array}{lll}1 & 1 & 1\end{array}\right],\left[\begin{array}{lll}1 & -1 & -1\end{array}\right]$ and $\left[\begin{array}{lll}-1 & 1 & -1\end{array}\right]$ in this frame. Near each body diagonal we assume six equilibrium directions for a $\mathrm{N}-\mathrm{D}$ bond, which are reached by a $\pm \varphi$ rotation about $X, Y$, and $Z$ axes. These positions are denoted by two indices $i$ and $j$, of which $i$ refers to one of the four body diagonals and $j$ to the six equilibrium directions near it. The probability of finding a certain deuteron at that site is denoted by $p_{i j}$.

The rate of rotation, which carries the certain deuteron from the vicinity of one body diagonal $i$ near to another diagonal $i^{\prime}$, is denoted by $W_{3} / 24$ (also in a rotation the three other deuterons of $\mathrm{ND}_{4}^{+}$change positions among those described above, but we just follow the position of one deuteron). The backward rotation is assumed to occur at the same rate. The jumping between the six directions near one body diagonal, at the rate $r / 6$, is assumed to be independent of the body diagonal $i$ and of the initial and final directions $j$ and $j^{\prime}$. Now we can write differential equations for the probabilities $p_{i j}$ of finding the certain deuteron at different positions $i j$, for example,

$$
\frac{d p_{11}}{d t}=-\left(\frac{3}{4} W_{3}+\frac{5}{6} r\right) p_{11}+\frac{1}{6} r \sum_{j=2}^{6} p_{1 j}+\frac{1}{24} W_{3} \sum_{i=2}^{4} \sum_{j=1}^{6} p_{i j} .
$$

Similar expressions can be written for the other probabilities. It is assumed that initially the deuteron is at the position 11 . Then $p_{11}(0)=1$ while all the remaining initial probabilities vanish so that $p_{i j}(0)=0$ for $i, j \neq 1$. By symmetry it can be concluded that the five probabilities $p_{i j}(j=2-6)$ are mutually equall and also the 18 quantities $p_{i j}(i=2-4$ and $j$ $=1-6)$ are mutually equal. Then Eq. (1) and similar ones for other independent probabilities reduce to 


$$
\begin{aligned}
& \frac{d p_{11}}{d t}=-\left(\frac{3}{4} W_{3}+\frac{5}{6} r\right) p_{11}+\frac{5}{6} r p_{12}+\frac{3}{4} W_{3} p_{21}, \\
& \frac{d p_{12}}{d t}=\frac{1}{6} r p_{11}-\left(\frac{3}{4} W_{3}+\frac{1}{6} r\right) p_{12}+\frac{3}{4} W_{3} p_{21}, \\
& \frac{d p_{21}}{d t}=\frac{1}{24} W_{3} p_{11}+\frac{5}{24} W_{3} p_{12}-\frac{1}{4} W_{3} p_{21} .
\end{aligned}
$$

These have the solution

$$
\begin{aligned}
& p_{11}=\frac{1}{8} e^{-W_{3} t}+\frac{5}{6} e^{-\left[(3 / 4) W_{3}+r\right] t}+\frac{1}{24} \\
& p_{12}=\frac{1}{8} e^{-W_{3} t}-\frac{1}{6} e^{-\left[(3 / 4) W_{3}+r\right] t}+\frac{1}{24}=p_{13}=\cdots=p_{16}, \\
& p_{21}=-\frac{1}{24} e^{-W_{3} t}+\frac{1}{24}=p_{22}=p_{23}=\cdots=p_{46} .
\end{aligned}
$$

For other initial conditions exactly the same occupation probabilities are obtained but with different subindices.

The deuteron spin-lattice relaxation is dominated by the quadrupole coupling. The $\Delta m= \pm 1$ and \pm 2 transitions are related to the functions

$$
B_{i j}^{(1)}=(3 / 8) \omega_{Q} \sin \theta_{i j} \cos \theta_{i j} \exp \left(-\iota \phi_{i j}\right)
$$

and

$$
B_{i j}^{(2)}=(3 / 16) \omega_{Q} \sin ^{2} \theta_{i j} \exp \left(-\iota 2 \phi_{i j}\right),
$$

respectively. The strength of the quadrupole interaction is given by $\omega_{Q}=e^{2} q Q / \hbar$ and the polar angles $\theta_{i j}$ and $\phi_{i j}$ define the orientation of the N-D vector $i j$ in the laboratory frame with the $Z$ axis parallel to the external magnetic field. The initial deuteron relaxation rate equals $1 / T_{1}=k_{1}+2 k_{2}$, where the $\Delta m= \pm 1$ and \pm 2 transition rates are ${ }^{23}$

$$
k_{\mu}=\left|M_{\mu}\right|^{2} \int_{-\infty}^{+\infty} C^{(\mu)}(t) e^{-i \mu \omega_{0} t} d t
$$

The spin matrix elements are $M_{1}=\sqrt{2}$ and $M_{2}=2$. The correlation function $C^{(\mu)}(t)$ is calculated for the both $\mu$ values 1 and 2 as (for brevity the index $\mu$ is dropped for a while)

$$
\begin{aligned}
C(t)= & \frac{1}{24}\left[B_{11}^{*}\left(p_{11} B_{11}+p_{12} \sum_{j=2}^{6} B_{1 j}+p_{21} \sum_{i=2}^{4} \sum_{j=1}^{6} B_{i j}\right)\right. \\
& +B_{12}^{*}\left(p_{11} B_{12}+p_{12} \sum_{\substack{j=1 \\
j \neq 2}}^{6} B_{1 j}+p_{21} \sum_{i=2}^{4} \sum_{j=1}^{6} B_{i j}\right) \\
& \ldots \\
& \left.+B_{46}^{*}\left(p_{21} \sum_{i=1}^{3} \sum_{j=1}^{6} B_{i j}+p_{12} \sum_{j=1}^{5} B_{4 j}+p_{11} B_{46}\right)\right] .
\end{aligned}
$$

Here $1 / 24$ is the probability of finding the deuteron under consideration at any of the 24 equilibrium positions and each line of Eq. (5) represents one equilibrium position as the initial position (there are altogether 24 lines). The expressions having $p_{21}$ as the multiplier in Eq. (5) contain $18 B_{i j}$ quantities on each line. When the occupation probabilities (3) are inserted into Eq. (5) and only the time-dependent terms retained, we obtain

$$
\begin{aligned}
C^{(\mu)}(t)= & \omega_{Q}^{2}\left[\frac{1}{24}\left(3 S_{0}^{(\mu)}+3 S_{1}^{(\mu)}-S_{2}^{(\mu)}\right) e^{-W_{3} t}+\frac{1}{6}\left(5 S_{0}^{(\mu)}\right.\right. \\
& \left.\left.-S_{1}^{(\mu)}\right) e^{-\left[(3 / 4) W_{3}+r\right] t}\right],
\end{aligned}
$$

with

$$
\begin{aligned}
& S_{0}=\frac{1}{24} \sum_{i=1}^{4} \sum_{j=1}^{6}\left|B_{i j}\right|^{2}, \\
& S_{1}=\frac{1}{24} \sum_{\substack{i=1 \\
j}}^{4} \sum_{\substack{j, j^{\prime}=1 \\
j \neq j^{\prime}}}^{6} B_{i j}^{*} B_{i j^{\prime}}, \\
& S_{2}=\frac{1}{24} \sum_{\substack{i, i^{\prime}=1 \\
i \neq i^{\prime}}}^{4} \sum_{j, j^{\prime}=1}^{6} B_{i j}^{*} B_{i^{\prime} j} .
\end{aligned}
$$

When Eq. (6) is inserted into Eq. (4) and the Fourier transform calculated, the following initial relaxation rate is obtained:

$$
\begin{aligned}
R_{D}= & \frac{1}{T_{1}}=\frac{1}{6} \omega_{Q}^{2}\left[\left(3 S_{0}^{(1)}+3 S_{1}^{(1)}-S_{2}^{(1)}\right) J\left(\omega_{0}, W_{3}\right)+4\left(5 S_{0}^{(1)}\right.\right. \\
& \left.-S_{1}^{(1)}\right) J\left(\omega_{0}, \frac{3}{4} W_{3}+r\right)+4\left(3 S_{0}^{(2)}+3 S_{1}^{(2)}\right. \\
& \left.-S_{2}^{(2)}\right) J\left(2 \omega_{0}, W_{3}\right)+16\left(5 S_{0}^{(2)}-S_{1}^{(2)}\right) \\
& \left.\times J\left(2 \omega_{0}, \frac{3}{4} W_{3}+r\right)\right]
\end{aligned}
$$

where, for example, $J(\omega, W)=W /\left(W^{2}+\omega^{2}\right)$.

Our numerical calculations for polycrystaline samples show that on average

$$
\begin{aligned}
& {\left[3 S_{0}^{(1)}+3 S_{1}^{(1)}-S_{2}^{(1)}\right]_{\text {powder }}=\left[3 S_{0}^{(2)}+3 S_{1}^{(2)}-S_{2}^{(2)}\right]_{\text {powder }},} \\
& {\left[5 S_{0}^{(1)}-S_{1}^{(1)}\right]_{\text {powder }}=\left[5 S_{0}^{(2)}-S_{1}^{(2)}\right]_{\text {powder }} .}
\end{aligned}
$$

By ignoring the nonexponentiality arising from the fact that in the absence of sufficiently fast spin diffusion the deuterons in differently oriented crystallites relax at somewhat different rates, the relaxation rate (8) becomes

$$
\begin{aligned}
R_{D}= & \frac{1}{6} \omega_{Q}^{2}\left\{[ 3 S _ { 0 } ^ { ( 1 ) } + 3 S _ { 1 } ^ { ( 1 ) } - S _ { 2 } ^ { ( 1 ) } ] _ { \text { powder } } \left[J\left(\omega_{0}, W_{3}\right)\right.\right. \\
& \left.+4 J\left(2 \omega_{0}, W_{3}\right)\right]+4\left[5 S_{0}^{(1)}-S_{1}^{(1)}\right]_{\text {powder }} \\
& \left.\times\left[J\left(\omega_{0}, \frac{3}{4} W_{3}+r\right)+4 J\left(2 \omega_{0}, \frac{3}{4} W_{3}+r\right)\right]\right\} .
\end{aligned}
$$

The first line is the contribution of $120^{\circ}$ reorientations. However, for most reorientations the angle becomes progressively different from $120^{\circ}$ when the deviation angle grows. The 


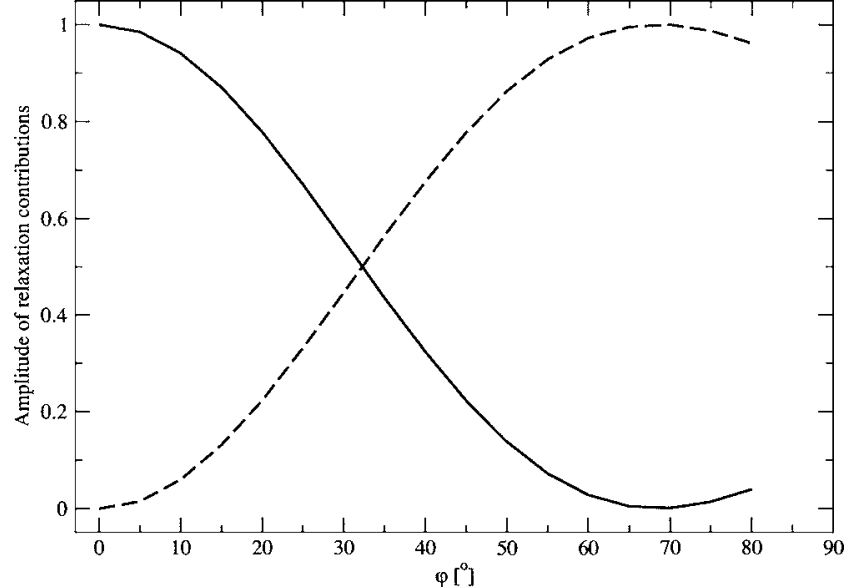

FIG. 1. Reduction of the deuteron relaxation rate amplitude via reorientational jumps of $\mathrm{ND}_{4}^{+}$as the function of the deviation angle $\varphi$ for polycrystalline samples (continuous line). The broken curve shows the dependence of the relaxation rate amplitude via limited jumps. The maximum value of 1 means the same relaxation efficiency as in the case of perfectly tetrahedral $120^{\circ}$ reorientations.

amplitude of this term, divided by the corresponding amplitude for the nondistorted structure $(\varphi=0)$, is shown in Fig. 1. The second line of Eq. (10) is the similarly normalized amplitude of the LJ contribution. Its dependence on the deviation angle $\varphi$ is also shown in Fig. 1.

If the deviation angle $\varphi$ vanishes so that the equilibrium directions of $\mathrm{N}-\mathrm{D}$ vectors are parallel to the body diagonals of the high-temperature cubic unit cell, then $5 S_{0}^{(\mu)}-S_{1}^{(\mu)}$ vanishes identically for both the $\mu$ values and Eqs. (8) and (10) reduce to earlier expressions describing the deuteron relaxation under $120^{\circ}$ reorientations about equivalent threefold axes. ${ }^{23}$ As Fig. 1 shows, the maximum value of this relaxation rate is reduced by $\mathrm{LJ}$ and the reduction grows with $\varphi$. The spectral density functions $J\left(\omega_{0}, \frac{3}{4} W_{3}+r\right)$ and $4 J\left(2 \omega_{0}, \frac{3}{4} W_{3}+r\right)$ become important when the deviation angle and thus $\left[5 S_{0}^{(1)}-S_{1}^{(1)}\right]_{\text {powder }}$ grows. If the rate $r$ of $\mathrm{LJ}$ is much larger than $W_{3}$, these functions represent the effect of $\mathrm{LJ}$. They can even become dominant at low temperatures. ${ }^{16,17}$ These two relaxation channels evolve gradually to each other and the change of roles is complete in the present motional model, when $\varphi$ reaches the value $70.5^{\circ}$. This angle is the complement of the tetrahedral angle $109.5^{\circ}$ between the threefold axes of the $\mathrm{ND}_{4}^{+}$. Such a reduction of the hightemperature maximum was already suggested in an early study on methyl groups, when the hindering potential shows three pairs of double minima as a result of a sixfold component in the potential. ${ }^{24}$ The main aim of this work was to discuss the reduction in the relaxation efficiency via torsional oscillations about the equilibrium orientations, an effect which also causes a small additional reduction in the case of $\mathrm{ND}_{4}^{+}$ions but is beyond the scope of the derivation above.

Below the order-disorder transition temperature of $27.2 \mathrm{~K}$ it can be assumed that four of the 24 equilibrium $\mathrm{N}-\mathrm{D}$ directions, say, 11, 21, 31, and 41, become preferred in the sense that it is more probable to find the corresponding positions occupied by the $\mathrm{ND}_{4}^{+}$deuterons than the remaining positions. This means that we have different transition rates for jumps to the preferred position $\left(r_{p r}\right.$ and $\left.W_{3 p r}\right)$ and from them $\left(r_{o p}\right.$ and $\left.W_{3 o p}\right)$. The derivation of the relaxation rate then becomes more complicated than the derivation described above, nevertheless we mention some of its consequences later on. It is important to note that the assumption of preferred positions is not in contradiction with neutron diffraction experiments ${ }^{7,25}$ if there is a sufficient number of differently ordered domains in the crystallites of the sample.

\section{Structure of spectra}

Deuteron NMR spectra are composed of doublets. These appear due to $\Delta m= \pm 1$ transitions within the level scheme obtained after diagonalization of the general Hamiltonian

$$
\mathcal{H}=\mathcal{H}_{Z}+\mathcal{H}_{T}+\mathcal{H}_{Q}+\overline{\mathcal{H}}_{D}
$$

composed of the Zeeman, tunneling, quadrupole, and secular dipolar terms for, in the case of $\mathrm{ND}_{4}^{+}$, the tetrahedral set of four deuterons. The Zeeman and tunneling Hamiltonians define zeroth order energy levels in terms of spin and space coordinates, respectively. The quadrupole Hamiltonian introduces the major perturbation due to relatively large coupling constant $C_{Q}$. The dipolar interaction only introduces some broadening of spectral components. Detailed discussion of features in the spectra of tunneling $\mathrm{ND}_{4}^{+}$ion can be found elsewhere. $^{26,27}$

We will restrict ourselves to what is essential for discussion here. The secular part of the quadrupole interaction vanishes for $A$ and $E$ symmetry species. Therefore, the $A$ and $E$ levels are shifted only in the second order. Such corrections are reduced by the increase of resonance or tunneling frequency to $C_{Q}^{2} / \nu_{0}$ or $C_{Q}^{2} / \nu_{t}$, respectively. The latter feature enables measurements of $\nu_{\mathrm{TA}}$ from separations between the doublets in the $A$ spectrum. ${ }^{26}$ The evaluation of the tunneling frequency up to about $8 \mathrm{MHz}$ from deuteron spectra is practically possible for $\mathrm{ND}_{4}^{+}$ions. For higher tunneling frequencies structureless narrow lines were calculated. The $T$ spectral components contribute a broad, rather flat background, particularly when their degeneracy is lifted.

The temperature dependence of the deuteron spectra was measured and analyzed in detail for $\left(\mathrm{ND}_{4}\right)_{2} \mathrm{SnCl}_{6} \cdot{ }^{26}$ Narrow spectra, albeit with clearly visible doublet structure, were observed. Doublet separations, increasing on increasing temperature, are inversely proportional to the tunneling frequency. The tunneling frequency $\nu_{\mathrm{TA}}$, obtained in this way, first shows a plateau at $7.5 \mathrm{MHz}$ below $15 \mathrm{~K}$ and decreases to zero near $50 \mathrm{~K}^{26,28}$ The $A$ spectra, discussed so far, are insensitive to ammonium reorientation. The $T$ components, which form a broad background at low temperatures, undergo motional narrowing. As a result they contribute to the central component with increasing intensity, which dominates in the spectrum and covers the $A$ doublet structure. Moreover, the tunneling frequency $\nu_{t}$ approaches zero in this temperature range. All those spectral components spread out by $\pm \nu_{t}$ at lower temperatures might be observed. However, measured spectra result from motional narrowing of the classical, rigid lattice spectrum. Figure 9 in Ref. 26 exhibits all features discussed above in the temperature dependence of the second moment. The measured second moment only in- 
cludes the central part of the spectrum. When all components at $\pm \nu_{t}$ would be taken into account the second moment would be equal to the value for a rigid ammonium ion.

The motional narrowing condition $\tau_{c} \delta \sim 1$, where we have a product of a correlation time $\tau_{c}$ and the half width $\delta$ of the spectrum, refers to homogeneous spectra of dipolarly coupled nuclei. As the deuteron spectrum is composed of many components with highly different distances from the center, the parameter $\delta$ refers now to a doublet separation. It means that doublets with a small separation will first undergo narrowing, i.e., at lower temperatures with correspondingly longer correlation times.

The narrowing process of doublets leads to the absorption spectrum $I(\omega)$,

$$
I(\omega)=\frac{(\omega-\bar{\omega})\left[2(\omega-\bar{\omega}) k+\Delta_{1} \delta \omega_{2}+\Delta_{2} \delta \omega_{1}\right]-\left(2 k+\frac{1}{2} \delta \omega\right)\left(\Delta_{1} \Delta_{2}-\delta \omega k-\delta \omega_{1} \delta \omega_{2}\right)}{\left(\Delta_{1} \Delta_{2}-\delta \omega k-\delta \omega_{1} \delta \omega_{2}\right)^{2}+\left[2(\omega-\bar{\omega}) k+\Delta_{1} \delta \omega_{2}+\Delta_{2} \delta \omega_{1}\right]^{2}},
$$

where $\omega_{1}=a$ and $\omega_{2}=-a$ define the frequencies of a symmetric doublet, while $\delta \omega_{1}$ and $\delta \omega_{2}$ are their natural line widths, respectively. The remaining quantities are $\delta \omega=\delta \omega_{1}+\delta \omega_{2}$, $\Delta_{1}=\omega-\omega_{1}, \Delta_{2}=\omega-\omega_{2}, \quad \bar{\omega}=\left(\omega_{1}+\omega_{2}\right) / 2$, and $k$ is the exchange rate. $^{29}$

Reorientation modulates the spin-dependent interaction. The corresponding correlation time $1 / W_{3}$, defined as the mean time between jumps, has the meaning of a lifetime of a given orientation. We will use the formula (12) in the following with the exchange rates understood as the lifetime of respective deuteron positions.

\section{EXPERIMENTAL}

\section{A. Experimental setup and procedure}

Deuteron relaxation was studied in a polycrystalline sample of fully deuterated ammonium hexachloroplatinate. The sample was previously used in inelastic neutron scattering (INS) experiments. ${ }^{30}$ The NMR experiments were performed with the Apollo (Techmag, USA) spectrometer, operating on the $7.04 \mathrm{~T} / 89 \mathrm{~mm}$ superconducting magnet and thus at the deuteron resonance frequency of $46 \mathrm{MHz}$. A low-

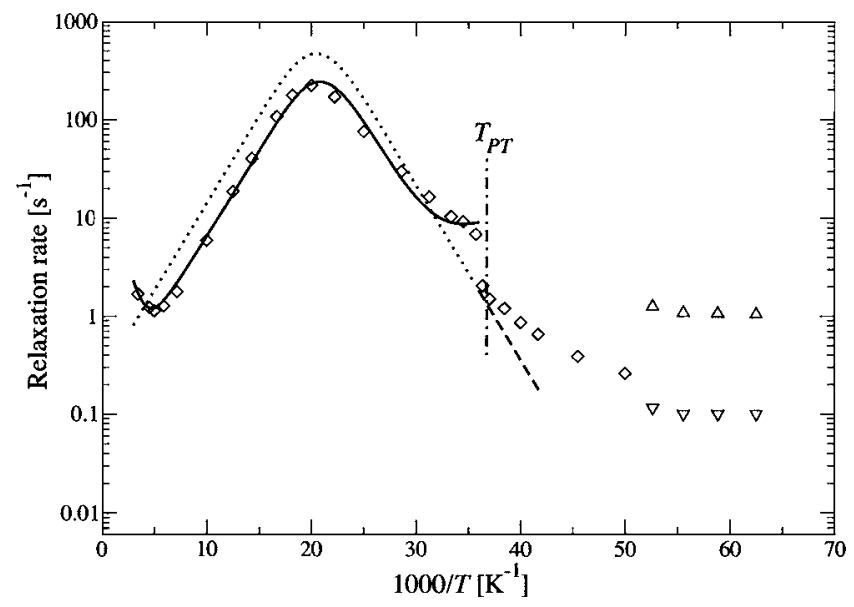

FIG. 2. Experimental deuteron relaxation rates in polycrystalline $\left(\mathrm{ND}_{4}\right)_{2} \mathrm{PtCl}_{6}$. The dotted and broken lines exclusively describe the relaxation via $120^{\circ}$ reorientations, while the continuous curve also includes the effect of limited jumps. The increase above $200 \mathrm{~K}$ arises from the motion of $\mathrm{PtCl}_{6}^{2-}$ anions. temperature NMR probe was placed inside the Oxford CF1200 continuous flow cryostat, and the temperature was regulated by the Oxford CT503 temperature controller. The temperature accuracy and stability were within $\pm 0.1 \mathrm{~K}$ in the whole temperature range.

The spin-lattice relaxation time was measured by the saturation-recovery method. An aperiodic ten-pulse saturating sequence was followed by $2 \mu$ s read pulse appearing after a variable time delay. The amplitude of the magnetization, recovered during the delay, was determined by recording the free induction decay. This was reasonable since the deuteron resonance spectrum remained narrow down to liquid helium temperatures, the width at the half height being only a few kilohertz at $5 \mathrm{~K}$. A satisfactory $S / N$ ratio was achieved by accumulating a number of signals. After the Fourier transform of the free induction decay, phase and baseline corrections, the signal intensity was determined by integration of the whole spectrum.

Typically 25 delays, covering the range from 0 to about $3 T_{1}$, were used to determine the magnetization recovery. An additional data point was measured at a time longer than $5 T_{1}$, in order to precisely determine the equilibrium magnetization. This approach was found to improve the accuracy of the three-parameter single-exponential fit to the data. Fits with two exponentials were necessary at lower temperatures, which lead to two characteristic rates and the corresponding weights.

\section{B. Experimental results and discussion-relaxation}

Figure 2 shows the spin-lattice relaxation rate of deuterons in $\left(\mathrm{ND}_{4}\right)_{2} \mathrm{PtCl}_{6}$ as a function of inverse temperature. A prominent maximum is seen near $50 \mathrm{~K}$. This maximum is the classical one corresponding to the condition $\omega_{0} \tau_{c}=\omega_{0} / W_{3}$ $\approx 1$. Increasing relaxation rate above $200 \mathrm{~K}$ can be explained by assuming that the electric field gradient at the deuteron site is modulated by the motion of $\mathrm{PtCl}_{6}^{2-}$ ions ${ }^{31}$ with an activation energy of about $8.5 \mathrm{~kJ} / \mathrm{mol}$. The same activation energy also fits the high temperature relaxation times of protons in $\left(\mathrm{NH}_{4}\right)_{2} \mathrm{PtCl}_{6}$, ${ }^{32}$ when the small contribution of spinrotational interaction to proton relaxation is included. ${ }^{33,34}$ The magnetic dipolar and spin-rotational interactions are 
more effective in relaxing protons than deuterons. Therefore, the motion of $\mathrm{PtCl}_{6}^{2-}$ ions has to be coupled to deuterons by electric quadrupole interaction.

We apply Eq. (10) to describe the experimental data between 27.2 and $200 \mathrm{~K}$. It is presented by the dotted line in Fig. 2, obtained with the parameters $\omega_{Q}=2 \pi \times 1.8 \times 10^{5} \mathrm{~s}^{-1}$, the activation energy $3.4 \mathrm{~kJ} / \mathrm{mol}$, the corresponding preexponential factor $W_{30}=2.4 \times 10^{12} \mathrm{~s}^{-1}$ in the Arrhenius equation, and the deviation angle $\varphi=0^{\circ}$. The calculated maximum value of the relaxation rate equal $470 \mathrm{~s}^{-1}$ is clearly too large. Furthermore, the dotted curve falls through the experimental data near $32 \mathrm{~K}$ and stays below them down to low temperatures. The reduction of the relaxation rate cannot be related to any tunnel splitting larger or roughly equal to $\omega_{0}$ because the splitting near $50 \mathrm{~K}$ is already negligible. Therefore, we explore the contribution of LJ, with an adjustable deviation angle $\varphi$. The continuous curve, corresponding to $\varphi=32^{\circ}$, fits the experimental points already much better, also between 28 and $35 \mathrm{~K}$. This curve implies a definite temperature dependence for the rate $r$ of $\mathrm{LJ}$, which was provided by the activation energy $E_{a r}=1.0 \mathrm{~kJ} / \mathrm{mol}$ and the preexponential factor $r_{0}=2 \times 10^{12} \mathrm{~s}^{-1}$. It means that $r$ is about 400 times larger than $W_{3}$ at $50 \mathrm{~K}$. The maximum contribution to the relaxation rate from $\mathrm{LJ}$ is expected to appear near $14 \mathrm{~K}$. The order-disorder transition and the onset of tunneling rotation complicate the situation in this region and Eqs. (8) and (10) are no more valid.

The deviation angle $32^{\circ}$ means an angle of $26.0^{\circ}$ between the individual equilibrium $\mathrm{N}-\mathrm{D}$ directions and the nearest $\mathrm{Pt}-\mathrm{N}$ vector. This result does not differ so much from the value of $20.9^{\circ}$, obtained by neutron diffraction. ${ }^{25}$

In order to understand the experimental data below the transition temperature of $27.2 \mathrm{~K}$ we have carried out some calculations for a model, in which the rate of the LJ to the preferred position, $r_{p r}$, differs from that for jumps from the preferred position $r_{o p}$. The corresponding rates for the practically $120^{\circ}$ rotations are denoted by $W_{3 p r}$ and $W_{3 o p}$, respectively. The amplitude of the term corresponding to the second line of Eq. (10) will have an additional multiplicative factor, approximately proportional to $r_{o p} / r_{p r}$. If $r_{p r}$ is much larger than the other rates, then this term, which represents the effect of LJ, has a vanishingly small amplitude. On the other hand the amplitude of the reorientation term, that is the first line of Eq. (10), will retain its maximum value consistent with $\varphi=0^{\circ}$. Actually in the ordered structure all the reorientations are perfectly tetrahedral. The rate $W_{3}$ is replaced by $W_{\text {eff }}=W_{3 p r} / 6+5 W_{3 o p} / 6$, because only the fraction $1 / 6$ of $120^{\circ}$ rotations are towards the preferred positions (the same holds for LJ). Thus there should be two kinds of changes in the relaxation rate at the transition temperature:

(i) The contribution of LJ is dramatically reduced as far as $r_{p r}$ is much larger than the three other rates (the experimental data suggest that this change is rather abrupt).

(ii) The effective rate of approximately $120^{\circ}$ rotations, $W_{3 p r} / 6+5 W_{3 o p} / 6$, may not be a smooth continuation of $W_{3}$ to temperatures below $27.2 \mathrm{~K}$.

The broken curve below the transition temperature in
Fig. 2 assumes that $r_{p r}$ becomes by far the largest rate at $27.2 \mathrm{~K}$ and $W_{\text {eff }}$ just below $27.2 \mathrm{~K}$ is equal to $W_{3}$ just above the transition temperature. The activation energy is kept constant. Therefore the broken curve is a continuation of the dotted curve to temperatures below $27.2 \mathrm{~K}$. In this way a rather good fit is obtained also between 27.2 and $25 \mathrm{~K}$.

Large-angle reorientations, described by a constant activation energy, start to become ineffective below $25 \mathrm{~K}$ relative to incoherent tunneling and therefore the broken curve deviates clearly from the experimental points. At still lower temperatures the relaxation becomes nonexponential and the rates of the fast and slowly relaxing components differ by an order of magnitude.

We consider various reasons for this nonexponentiality. At first there is a possibility for a level crossing. If the tunneling frequency $\nu_{\mathrm{TA}}$ is equal to $\nu_{0}$ or $2 \nu_{0}$, then the rate of deuteron relaxation should be increased. Such a level crossing might be possible in $\left(\mathrm{ND}_{4}\right)_{2} \mathrm{PtCl}_{6}$, since the estimates for $\nu_{\mathrm{TA}}$ vary between 40 and $200 \mathrm{MHz}$. However, earlier experiments on $\left(\mathrm{ND}_{4}\right)_{2} \mathrm{SnCl}_{6}$, showed that the increase in the relaxation rate is quite small and very difficult to observe. ${ }^{28}$ Therefore a more likely explanation is needed.

Another possible reason might be a tunneling splitting clearly larger than the resonance frequency. This could be true, if the tunneling frequency $\nu_{\mathrm{TA}}$ is near the higher estimate of $200 \mathrm{MHz}$ below $20 \mathrm{~K}$. The relaxation of the $T$ species $\mathrm{ND}_{4}^{+}$ions would then take place predominantly via transitions involving energy changes $\pm \omega_{0}$ or $\pm 2 \omega_{0}$, while that of the $A$ (correspondingly $E$ ) species ions would occur via transitions involving changes $\pm\left(\omega_{\mathrm{TA}} \pm \omega_{0}\right)$ or $\pm\left(\omega_{\mathrm{TA}} \pm 2 \omega_{0}\right)$. Therefore, the $T$ species ions should relax much faster than the $A$ and $E$ species ions if $\omega_{\mathrm{TA}}, \omega_{\mathrm{ET}} \gg \omega_{0}$. If reorientation and incoherent tunneling were the only active motions in the deuteron relaxation, then the rate of both the fast and slowly relaxing components should decrease monotonously with temperature. ${ }^{14}$ Furthermore, the $A$ and $E$ species ions should relax even more slowly because of the large tunneling frequency in the denominator of the spectral density function. However, experimentally the rate of the fast component is observed to grow near $20 \mathrm{~K}$ when temperature is lowered, and that of the slow component does not show any drastic decrease (there is a small step related to the fact that above $20 \mathrm{~K}$ the data were fitted with one exponential but below that temperature with two exponentials). The only explanation we can imagine is the contribution of LJ. ${ }^{14}$ Estimates based on a more general motional model, with different rates for rotations and $\mathrm{LJ}$ to the preferred and nonpreferred orientations, suggest that a contribution of the desired magnitude is obtained when $r_{o p} / r_{p r}$ is about 0.003 . Also LJ as the dominant relaxation mechanism relax $T$ species ions more effectively than the $A$ and $E$ ions. ${ }^{17}$ Of course this explanation requires that at least a part of the induction signal, related to the $T$ species ions, is observed below $20 \mathrm{~K}$, in spite of the fact that a considerable part of it is broadened beyond observation.

Ordered ammonium ions forming differently ordered domains provide still another possible explanation for the nonexponentiality at low temperatures. In this case the tunneling frequency can be of the same magnitude or smaller than the resonance frequency. Inside the domains the relaxation rate 


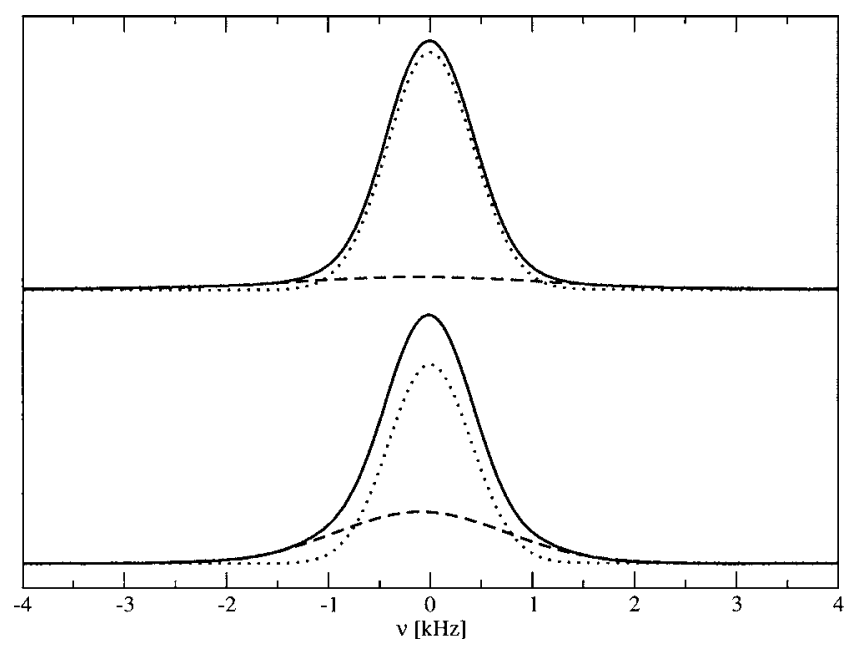

FIG. 3. Examples of experimental spectra (continuous line) at 27 (top) and $9 \mathrm{~K}$ (bottom) with the narrow (dotted line) and broad (broken line) components.

can be explained by Eq. (10) by continuing the broken curve in Fig. 2 to lower temperatures. Below $25 \mathrm{~K}$ the dominant motion is incoherent tunneling with a very small "activation energy," which means a slower decrease of the broken curve with temperature. LJ do not have any effect, which means that the ratio $r_{o p} / r_{p r}$ has to be even smaller than 0.003 . The fast relaxing component below $20 \mathrm{~K}$ would be related to domain boundaries with $r_{o p} / r_{p r}$ roughly equal to 0.003 . Since nonexponentiality disappears above $20 \mathrm{~K}$, the relaxation rates of the two components have to approach each other. As mentioned above, the rate of the fast component actually decreases somewhat when temperature is raised above $20 \mathrm{~K}$. This can be understood, if the jump rate $r_{p r}$ is larger than $\omega_{0}$ already at $15 \mathrm{~K}$ and above it, so that $\mathrm{LJ}$ are in the fast motion regime. These requirements are consistent with the temperature dependence of the jump rate $r$ used in simulating the experimental data of Fig. 2 above the transition temperature.

Our relaxation data are not sufficient to decide if the nonexponentiality below $20 \mathrm{~K}$ is related to a large tunneling frequency $\nu_{\mathrm{TA}} \gg \nu_{0}$ or if the existence of domains and domain boundaries is the dominant factor. However, the spectral experiments give some support for latter model.

\section{Experimental results and discussion spectra}

The spectra remain narrow in the whole temperature range, while two components were obtained below $55 \mathrm{~K}$ (Figs. 3-5). The temperature dependence of the half width of the spectra is shown in Fig. 4. The width of narrow component grows slightly on decreasing temperature and exhibits a steplike increase from $0.75 \mathrm{kHz}$ to about $1 \mathrm{kHz}$ slightly above PT. A broader component appears at about $55 \mathrm{~K}$. Its width increases up to about $3.5 \mathrm{kHz}$ also slightly above PT and drops down to its initial value of $2 \mathrm{kHz}$ below $20 \mathrm{~K}$.

Relative contributions of narrow and broad components are shown in Fig. 5. The contribution $S_{b}$ of the broad component increases up to about $40 \%$ just above PT, drops down to about $15 \%$ at PT, and approaches a plateau of about $35 \%$ below $20 \mathrm{~K}$.

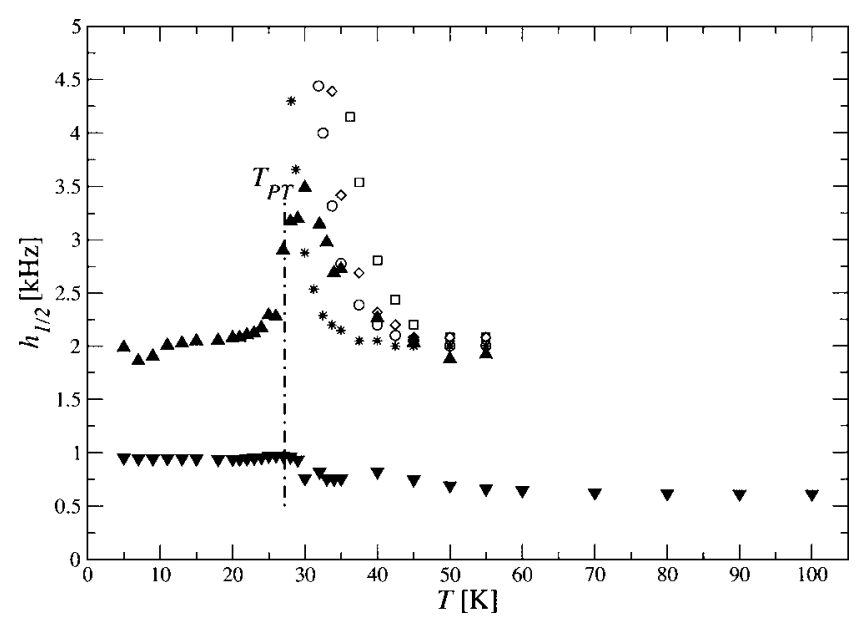

FIG. 4. Temperature dependence of the half width of the narrow $(\boldsymbol{\nabla})$ and broad ( $\mathbf{\Lambda})$ spectral components. The half widths calculated from Eq. (12) are shown for the doublet separations $2 a=40(*), 100(\bigcirc), 135(\diamond)$, and $200 \mathrm{kHz}(\square)$ in the temperature range between 30 and $55 \mathrm{~K}$.

There are two important points to be taken into account in discussing the temperature dependence of spectra: the range where the tunneling splitting is significant and PT temperature. Calculations indicate that the tunneling frequency of $\mathrm{ND}_{4}^{+}$in $\left(\mathrm{ND}_{4}\right)_{2} \mathrm{PtCl}_{6}$ amounts to about $10 \mathrm{MHz}$ at $35 \mathrm{~K}$ and is larger at lower temperatures.

Combined reorientations and LJ are very efficient in narrowing spectra as is seen at high temperatures. A broader component appears at about $55 \mathrm{~K}$. Its contribution grows linearly up to about $40 \%$ when the temperature is lowered down to about $30 \mathrm{~K}$. We attribute it to a sort of seeds of the ordered phase. LJ are therefore largely suppressed and a fraction of ammonium ions perform reorientations only.

We may also treat the spectrum as motionally narrowed classical one. For some simulations we take Eq. (12) and use for the exchange rate $k$ the reorientation frequency $W_{3}$ as obtained from the fit of Eq. (10) to the relaxation rates. From the broad deuteron spectrum of rigid $\mathrm{ND}_{4}^{+}$ions, spreading over the range $\pm 135 \mathrm{kHz}$, we have selected some representative doublet positions. The calculated half widths for different doublet separations for the temperature range of

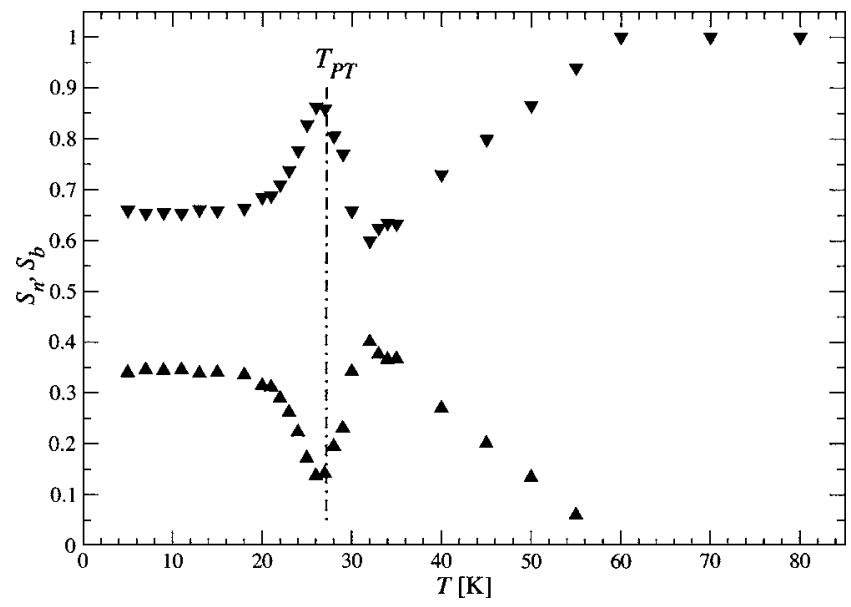

FIG. 5. Temperature dependence of the relative weights of the narrow ( and broad ( $\mathbf{\Lambda})$ spectral components. 
$30-50 \mathrm{~K}$ fall above (large separation) and below (small separation) the experimental points in Fig. 4. This is understandable because the experimental data represent the average half width for many doublets with separations between 1 and $135 \mathrm{kHz}$.

At about $35 \mathrm{~K}$ we enter the quantum-rotor temperature range. We expect spectra composed of a narrow $A$ line and motionally narrowed $T$ components. The latter come from the $T$ components with $a<20 \mathrm{kHz}$ which start to broaden at lower temperatures. Finally all $T$ components disappear from the center of the spectrum. In result $A$ components emerge as the dominant part of the observed spectrum and the line width equals $2 \mathrm{kHz}$.

The weight of the broad component shows a minimum around the PT temperature. The process of ordering is a very dynamic one, for example, regions of diverse mobility, i.e., ordered domains and transition regions between them, may propagate in space. Finally at the lowest temperatures we have $35 \%$ of $\mathrm{ND}_{4}^{+}$ions in ordered domains. The weights of spectral components represent the measured part of spectrum. We must be aware of existence of missing sidebands. At temperatures below about $50 \mathrm{~K}$ rotational sidebands move in, while below about $35 \mathrm{~K}$ tunneling sidebands move out.

\section{CONCLUSION}

We have presented here three features observed by deuteron NMR around the order-disorder PT in $\left(\mathrm{ND}_{4}\right)_{2} \mathrm{PtCl}_{6}$. There is a discontinuity in the relaxation rate at PT and also the width and relative weights of the two components in the spectrum change significantly around PT. The changes are, in the first place, caused by changes in the rotational and LJ rates, which seem to be partly discontinuous due to the occurrence of the order-disorder PT.

The presented motional model for the deuteron relaxation, leading to Eqs. (8) and (10), describes the experimental data quite well between 27.2 and $200 \mathrm{~K}$ (Fig. 2). The obtained agreement is based on the assumption that the equilibrium directions of the $\mathrm{N}-\mathrm{D}$ vectors are not parallel to the $\mathrm{Pt}-\mathrm{N}$ directions but deviate from them. Jumping between the six orientations near a certain $\mathrm{Pt}-\mathrm{N}$ vector is much faster than large-angle reorientations and reduces the relaxation efficiency of the $120^{\circ}$ reorientations. The observed reduction by about $53 \%$ at the maximum near $50 \mathrm{~K}$ fits best the deviation angle $\varphi=32^{\circ}$, corresponding to the angle of $26.0^{\circ}$ between each $\mathrm{N}-\mathrm{D}$ equilibrium direction and the nearest $\mathrm{Pt}-\mathrm{N}$ vector. Earlier results for this angle, based on the maximum value of the relaxation rate via LJ, were clearly smaller than the corresponding INS results. ${ }^{16,17}$ The reason for such large differences may be found in the fact that the jump rates rates $r_{p r}$ and $r_{o p}$ in the ordered structure (to the preferred position and from it, respectively), are clearly different, causing an additional reduction in the LJ relaxation rate, roughly proportional to $r_{o p} / r_{p r}$.

LJs start to directly contribute to the relaxation rate below $50 \mathrm{~K}$. The theoretical curve deviates slightly from the experimental data between 27.2 and $50 \mathrm{~K}$. A better agreement could very likely be obtained, if the jump rates $r_{p r}$ and $r_{o p}$ (similarly $W_{3 p r}$ and $W_{3 o p}$ ) are allowed to differ from each other already above $27.2 \mathrm{~K}$. Such an extended model would, however, contain many new parameters so that definite conclusions would be difficult to draw. Just below the transition temperature the LJ contribution to the relaxation rate is vanishingly small because of the additional reduction factor $r_{o p} / r_{p r}$. In this case, in contrast to structural PTs, the discontinuity in observed relaxation rate is related to the disappearence of LJ. Therefore the deuteron relaxation is dominated by $120^{\circ}$ reorientations or incoherent tunneling below $25 \mathrm{~K}$.

For the nonexponentiality of the deuteron relaxation below $20 \mathrm{~K}$ there are at least two possible explanations. One is based on the assumption that the tunneling frequency $\nu_{\mathrm{TA}}$ $\gg \nu_{0}$. The fast and slow components are related to the $T$ and $A+E$ species ammonium ions, respectively. LJs are needed to account for the increase especially in the fast rate below $20 \mathrm{~K}$. The other model assumes a tunneling frequency equal or smaller than $\nu_{0}$ and the existence of ordered domains of ammonium ions and domain boundaries. The slow component would be related to domains and the fast one to boundaries. The dominant relaxation mechanism in the boundaries would be LJ, while reorientation and incoherent tunneling would dominate in domains. Relaxation experiments are not sufficient to determine which model is better but spectral data seem to prefer the latter one.

${ }^{1}$ T. Matsuo, A. Inaba, O. Yamamuro, and N. Onoda-Yamamuro, J. Phys.: Condens. Matter 12, 8595 (2000).

${ }^{2}$ M. Prager, W. Press, A. Heidemann, and C. Vettier, J. Chem. Phys. 80, 2777 (1983).

${ }^{3}$ R. D. Weir and E. F. Westrum, Jr., J. Chem. Thermodyn. 23, 653 (1991).

${ }^{4}$ J. Pelzl and C. Dimitropoulos, Z. Naturforsch., A: Phys. Sci. 49, 232 (1995).

${ }^{5}$ H. Muraoka, T. Matsuo, and Y. Kume, Solid State Commun. 93, 529 (1995).

${ }^{6}$ R. D. Weir and E. F. Westrum, Jr., J. Chem. Thermodyn. 34, 133 (2002).

${ }^{7}$ T. Matsuo, Pure Appl. Chem. 75, 913 (2003).

${ }^{8}$ K. Vogt and W. Prandl, J. Phys. C 16, 4753 (1983).

${ }^{9}$ Th. Brückel, W. Prandl, K. Vogt, and C. M. Z. Zeyen, J. Phys. C 17, 4071 (1984)

${ }^{10}$ Y. Kume and T. Asaji, J. Mol. Struct. 345, 145 (1995).

${ }^{11}$ A. Birczyński, Z. T. Lalowicz, Z. Olejniczak, G. Stoch, and B. Petelenz, Chem. Phys. Lett. 339, 229 (2001).

${ }^{12}$ P. Filipek, Z. T. Lalowicz, Z. Olejniczak, and A. Birczyński, Chem. Phys. Lett. 367, 55 (2003).

${ }^{13}$ E. E. Ylinen, P. Filipek, M. Punkkinen, and Z. T. Lalowicz, Physica B 357, 456 (2005).

${ }^{14}$ A. Birczyński, Z. T. Lalowicz, M. Punkkinen, and A. M. Szymocha, Chem. Phys. 327, 373 (2006).

${ }^{15}$ Z. T. Lalowicz, M. Punkkinen, Z. Olejniczak, A. Birczyński, and U. Haeberlen, Solid State Nucl. Magn. Reson. 22, 373 (2002).

${ }^{16}$ L. P. Ingman, E. Koivula, M. Punkkinen, E. E. Ylinen, and Z. T. Lalowicz, Physica B 162, 281 (1990).

${ }^{17}$ M. Punkkinen and E. E. Ylinen, Physica B 337, 111 (2003).

${ }^{18}$ M. Prager and A. Heidemann, Chem. Rev. (Washington, D.C.) 97, 2933 (1997).

${ }^{19}$ M. Prager, A. M. Raaen, and I. Svare, J. Phys. C 16, L181 (1983).

${ }^{20}$ T. P. Das, J. Chem. Phys. 25, 896 (1956).

${ }^{21}$ T. P. Das, J. Chem. Phys. 27, 763 (1957).

${ }^{22}$ A. Birczyński, Z. T. Lalowicz, and Z. Lodziana, Chem. Phys. 299, 113 (2004).

${ }^{23}$ Z. T. Lalowicz, M. Punkkinen, A. H. Vuorimäki, E. E. Ylinen, A. Detken, and L. P. Ingman, Solid State Nucl. Magn. Reson. 8, 89 (1997). 
${ }^{24}$ C. S. Johnson, Jr., J. Magn. Reson. (1969-1992) 24, 63 (1976).

${ }^{25}$ O. Yamamuro, K. Okishiro, T. Matsuo, N. Onoda-Yamamuro, K. Oikawa, T. Kamiyama, Y. Kume, and F. Izumi, J. Chem. Phys. 107, 8004 (1997).

${ }^{26}$ L. P. Ingman, E. Koivula, Z. T. Lalowicz, M. Punkkinen, and E. E. Ylinen, J. Chem. Phys. 88, 58 (1988).

${ }^{27}$ Z. T. Lalowicz, Z. Naturforsch., A: Phys. Sci. 43, 895 (1988).

${ }^{28}$ L. P. Ingman, E. Koivula, Z. T. Lalowicz, M. Punkkinen, and E. E. Ylinen, Z. Phys. B: Condens. Matter 81, 175 (1990).
${ }^{29}$ A. Carrington and A. D. McLachlan, Introduction to Magnetic Resonance (Harper \& Row, New York, 1969), Chap. 12.

${ }^{30}$ M. Prager, P. Schiebel, and H. Grimm, J. Chem. Phys. 116, 10338 (2002).

${ }^{31}$ M. Bonori and M. Terenzi, Chem. Phys. Lett. 27, 281 (1974).

${ }^{32}$ I. Svare, A. M. Raaen, and G. Thorkildsen, J. Phys. C 11, 4069 (1978).

${ }^{33}$ L. Niémelä and E. Ylinen, Ann. Acad. Sci. Fenn., Ser. A6 307, 1 (1969).

${ }^{34}$ R. Ikeda and C. A. McDowell, Chem. Phys. Lett. 14, 389 (1972). 